\title{
Good intentions: drivers' decisions to engage with technology on the road and in a driving simulator
}

\author{
Katie J. Parnell ${ }^{1}\left[\right.$ (] Neville A. Stanton ${ }^{1} \cdot$ Katherine L. Plant ${ }^{1}$ \\ Received: 6 November 2017 / Accepted: 5 July 2018 / Published online: 13 July 2018 \\ (c) The Author(s) 2018
}

\begin{abstract}
Distraction-related accidents are, more often than not, due to the drivers' voluntarily engagement with a secondary task. Therefore, the strategic management of in-vehicle tasks and the drivers' decision to engage with them is an important aspect of the driver distraction phenomenon that needs to be addressed. While the consequences of distracting tasks are often assessed in settings where the risk of engaging is reduced (i.e., simulators), the drivers' decision to engage with secondary tasks is often ignored. This study assessed the drivers' decision to engage with secondary tasks using verbal protocols to provide insights into the drivers' intention within a naturalistic driving setting, on the road, and in a simulated driving environment. This enabled an understanding of when drivers engage with technological distractions, why they choose to do so, as well as how they may go about doing it. Different road types were found to differentially impact the drivers' intention to engage, as did the types of secondary tasks, with some tasks having an increased willingness to engage compared to others. Factors that increase and/or reduce the likelihood of engaging are presented. The decisions that drivers made to engage with secondary tasks in the simulator were found to correlate strongly to their decision to so on the road. This provides support for the use of simulators when assessing the drivers' decision to engage with secondary tasks. The effect of verbal protocols on the drivers' speed metrics was assessed to determine how they may have affected their driving performance.
\end{abstract}

Keywords Driver distraction $\cdot$ In-vehicle technology $\cdot$ Naturalistic decision making $\cdot$ Verbal protocol analysis

\section{Introduction}

The use of technology by drivers has long been a concern for safety practitioners and researchers who are aware of the distractive potential that secondary tasks have on the primary driving task (Brown et al. 1969; Stutts et al. 2001; Regan et al. 2008; Young and Regan 2007; WHO 2011). Device manufacturers have allowed the integration of technology within the vehicle and the development of portable technologies that can be brought into the vehicle. They are thus promoting the driver to self-regulate their interactions in line with their own motivations and intentions (Horrey and Lesch 2009; Lee 2014). The 100-car study (Dingus et al. 2006), a large naturalistic driving study, found distraction from

Katie J. Parnell

kp4g13@soton.ac.uk; k.parnell@soton.ac.uk

1 Transportation Research Group, Faculty of Engineering and the Environment, Boldrewood Innovation Campus, University of Southampton, Burgess Road, Southampton SO16 7QF, UK secondary tasks to be the most common form of inattention and wireless devices to be the most common secondary task that led to distraction-related events (e.g., crashes and nearcrashes). In a more recent analysis of crash reports, Beanland et al. (2013) found 70\% of distraction-related crashes to be voluntarily engaged by the driver. This suggests that distractions occur as a result of the individual making the considered decision to engage with secondary tasks, despite being aware of the risks. Although, it has also been suggested that drivers are skilled at self-regulating when they engage with secondary tasks if they can predict the upcoming demand (Tivesten and Dozza 2015). The Prioritise, Adapt, Resource, Regulate, Conflict (PARRC) model of distraction (Parnell et al. 2016) proposed 'Behavioural regulation' to be one of the five key factors in distraction from technological sources which reflects the self-management of attention, effort, attitudes and emotions to facilitate goal attainment in the development of distraction-related events.

To prevent drivers from engaging with distracting task, legislation has banned distracting activities and penalties are given to those caught engaging with tasks that could pose 
as distractions. The risks associated with distracting tasks are also communicated to drivers through media campaigns and road safety charities. Yet, a survey by Young and Lenné (2010) found that drivers are still willing to engage with distracting tasks, even if they know the risks of an accident occurring are increased. This suggests that the current methods targeting drivers may be not be wholly effective. There is an assertion that there needs to be a cultural shift in how we view accidents that should not seek to attribute blame towards individuals but should instead be more proactive in preventing such incidents from occurring. This can be done by looking towards the wider system in which the incident occurs (e.g., Reason 1990; Dekker 2002). Rather than focusing on the driver and their role in distraction, Salmon et al. (2012) highlighted the importance of reviewing the sociotechnical system within which driver distraction occurs. This is an avenue of research that has been followed up to determine the implications of applying the sociotechnical systems theory to driver distraction and how it may inform alternative and effective mitigation strategies (Tingvall et al. 2009; Young et al. 2013; Young and Salmon 2015; Parnell et al. 2016, 2017a, b).

Rather than being passive reactors to high levels of workload that exceed attentional resource capacity (e.g., Wickens 2002), it is now understood that drivers adapt their behaviour and attentional resources relative to the demands of the situation to alter the distractive effects of secondary tasks (e.g., Brookhuis et al. 1991; Haigney et al. 2000; Young and Stanton 2002, 2004; Rakauskas et al. 2004; Tivesten and Dozza 2015; Metz et al. 2015). Indeed, 'Adapt to demands' emerged as another key mechanism of distraction in the PARRC model (Parnell et al. 2016), which captured the prominence of the drivers' adaption of their behaviour to manage concurrent goals alongside the primary driving goal. A key development of the PARRC model was the recognition that distraction is related to the wider sociotechnical system. Central to this was the interaction between the PARRC mechanisms and their interdependence on each other in the emergence of driver distraction, as well as the underlying systemic causes. These underlying causal factors included: road infrastructure, other road users, traffic demand, environmental conditions, vehicle manufacturers and government policy (Parnell et al. 2016). This avenue of research suggested that the voluntary decisions of the driver to engage with distracting tasks may not be a direct result of the driver alone, but their complex relationship with the sociotechnical system within which the behaviour occurs. Yet, acknowledging the 'voluntary' aspect of distraction does highlight the importance of understanding the dynamics of the behaviour (Lee 2014) and the interacting factors, elements and conditions within the wider sociotechnical system (Tingvall et al. 2009; Young and Salmon 2015; Parnell et al. 2016, 2017a, b). The drivers' decision to be distracted is, therefore, an important component to understand (Lerner 2005; Horrey and Lesch 2009; Lee 2014; Tivesten and Dozza 2015; Metz et al. 2015).

\subsection{Naturalistic decision making}

Traditional decision-making theories suggest that an individual makes their choice from a set of alternatives using optimisation strategies generated from identifying multiple options, their probability and utility estimates. A more recent understanding in the domain suggests that decisions are actually based on intuition (Phillips et al. 2004), experience (Klein et al. 1986), biases and heuristics (Kahneman et al. 1982). The process of satisficing which relates to finding the first 'good enough' option rather than the best possible option (Simon 1957) has been realised when assessing decision makers in their naturalistic environment (Klein 1989). This phenomenon has also been linked to driver distraction in the minimum required attention theory (MiRA; Kircher and Ahlstom 2017) which incorporates the notion that driving is a satisficing and self-paced task (e.g., Boer and Hoedemaeker 1998; Summala 2007). Kircher and Ahlstrom (2017) deem drivers to be attentive when they are collecting 'enough' information from the road environment as is needed relative to the current demands, allowing the driver to adapt their behaviour and engagement with secondary tasks in line with the demands of the situation. This view of distraction suggests it to be heavily related to the situation in which it arises, which complements the findings that drivers adapt their behaviour in line with the situational demands (e.g., Haigney et al. 2000; Rakauskas et al. 2004; Brookhuis et al. 1991; Parnell et al. 2016). When assessing distraction, it is, therefore, important to account for the situational demands and the context surrounding the behaviour (Sharples et al. 2016). Yet, capturing driver behaviour in its naturalistic environment has its own challenges (Carsten et al. 2013).

\subsection{Experimental setting}

The road safety risks that distracted driving poses has meant that many studies that aim to observe the consequences of distraction have utilised driving simulators (e.g., Summala et al. 1998; Tsimhoni et al. 2004; Reimer 2009) or test-track facilities (e.g., Horrey and Lesch 2009; Ranney et al. 2005). These provide controlled and safe environments to trial new technologies and measure driving performance (Burnett 2008). Yet, it is difficult to validate simulation studies, and there have been few attempts to do so in the literature (Burnett 2008). Technological advancements have vastly improved driving simulation facilities, enhancing the fidelity and validity of experimental studies (e.g., Eriksson et al. 2017). This is to the advantage of distraction research that 
is often unsafe and unethical to perform on the road. Such facilities have enabled an understanding of the adverse consequences of secondary task engagement when the onset of the task is pre-determined and controlled within the experiment (e.g., Lansdown et al. 2004). Yet, if it is understood that distractions are largely voluntarily engaged by drivers (Beanland et al. 2013), then the pre-determined scenarios cannot be generalised to the scenarios, where drivers choose to engage with secondary tasks. They fail to account for any strategic engagement with the secondary task which may offset any adverse effects. For example, Lee and Strayer (2004) highlight the overestimation of risk attributed to older drivers engaging with hand held phones from studies that forced drivers to engage with the technology, when older drivers may not actually choose to engage with the phone at all when driving in their everyday lives.

The decision to engage with distractions has been explored under a range of experimental conditions, although this area of distraction has still attracted limited research in contrast to those who impose the onset of secondary tasks and ignore the active role of the driver. In a semi-structured interview study, Parnell et al. (2017a) asked drivers to rate their likelihood of engaging with a range of technological devices (mobile phone, sat-nav, infotainment system, hand-free phone) while detailing (in an open-ended discussion with the researcher) their intentions and reasoning why. They were asked to detail their hypothetical reasoning for their decision across different road environments that were found by Walker et al. (2013) to influence the drivers' situational awareness. This aimed to explore how the road context may influence the drivers' likelihood of engaging with different technological tasks using scenarios to elicit driver opinions and perceptions. This is a method that is valuable in encouraging people to think about a wide range of situations in a limited time period (Sharples et al. 2016). Inductive thematic analysis of the transcripts from the openended discussion generated a framework of the key themes that underlined the drivers stated likelihood of engaging with each of the technologies within the interview setting (Parnell et al. 2017a; Table 1).

The interview study highlighted the complexity of factors that influence the drivers' decision to engage with a secondary task, as interpreted from the drivers own perspective. Yet, the interviews were only able to capture the drivers' hypothetical reasoning for engaging with technologies while driving. They did not capture the context surrounding the interaction and how this may impact on intention. Holtzblatt and Jones (1993) highlight the importance of describing

Table 1 Thematic framework from Parnell et al. (2017a)

\begin{tabular}{|c|c|c|}
\hline Systems themes & Semantic subtheme & Example from interviews with drivers \\
\hline \multirow[t]{3}{*}{ Context } & Journey & $\begin{array}{l}\text { "if I am in a strange city, I would be less likely to mess around because I don't know where I am } \\
\text { going" }\end{array}$ \\
\hline & Road & "I think it would be situational dependent, just how busy is it? I think" \\
\hline & Task & $\begin{array}{l}\text { "It's stuff when I actually feel like I need to send a message quickly, so if I've agreed to come home at } \\
\text { a certain time and I'm running late for instance" }\end{array}$ \\
\hline \multirow[t]{4}{*}{ Driver } & Attitude of the driver & "I don’t see any problem with it personally whatsoever" \\
\hline & Influence of others & "the shame if you did something bad, that everyone would think you are so stupid" \\
\hline & Tendency & "I have been known to do that" \\
\hline & View of self/behaviour & "if I am stopped I generally am a little bit more naughty" \\
\hline \multirow[t]{5}{*}{ Infrastructure } & Illegality & "I usually hold it in a low position, so the police can't see" \\
\hline & $\begin{array}{l}\text { Perception of sur- } \\
\text { rounding environ- } \\
\text { ment }\end{array}$ & "for these roads and junctions, it would require a lot more concentration" \\
\hline & Road layout & "because to me a motorway, once you are on it, it is all moving in the same direction generally" \\
\hline & Road-related behaviour & "urban road I think is more busy as well so I think the more sort of decisions you've got to make" \\
\hline & Task-road relationship & $\begin{array}{l}\text { "Yeah it would be stilted, I would probably make the person on the phone aware, say hang on a min- } \\
\text { ute but I would probably sound not as engaged in the conversation" }\end{array}$ \\
\hline \multirow[t]{6}{*}{ Task } & Ability to complete & "because I've had the car for ages, I know where the switches are" \\
\hline & Complexity & $\begin{array}{l}\text { "if you have to unlock the phone screen or whatever etc., it is not as simple - well it is quite distract- } \\
\text { ing" }\end{array}$ \\
\hline & Desirability & $\begin{array}{l}\text { "I don't really use my phone very much anyway so it's never been something that I have felt I have } \\
\text { needed" }\end{array}$ \\
\hline & Duration & "if it's a long text you might not read it" \\
\hline & Engagement regulation & "I will always figure out what I'm going to listen to and set it going before I leave" \\
\hline & Interaction & "It's only one button to press, so that's not an issue" \\
\hline
\end{tabular}


behaviour in the context within which the behaviour is produced. A description of behaviour outside of its context is a description that is based on ways that it could or should be performed, but fails to capture the processes involved in the behaviour (Holtzblatt and Jones 1993). The knowledge that has been gained through exploring decisions in their natural environment, as opposed to in highly controlled laboratories, have developed understanding across many domains (Klein 2008). Therefore, the applicability of this framework to natural driving conditions needs to be addressed.

Research into the drivers' decision to engage with tasks when they are performing the task of driving have been limited, yet a study by Metz et al. (2011) conducted in a driving simulator compared the drivers' decision to engage, and consequently perform, in an externally paced secondary task and a self-paced task. They found that drivers utilised the self-paced task to adapt their visual processing of the driving task and the visual secondary tasks in line with the demands of the environment. Further work by Schömig and Metz (2013), in another driving simulator study, found evidence that drivers adapted their behaviour across three levels of situational awareness. Strategic adaption occurred when drivers made high level decisions about which situation to engage with a secondary task. Decision level adaption occurred when the driver decided to go ahead with interactions with secondary tasks in line with the demands of driving situation. Control level adaption occurs once the secondary task has been engaged with and drivers adapt driving behaviour on a momentary basis (e.g., by slowing down), or secondary tasks interactions (e.g., interrupted glances), to integrate the two tasks. When assessing the drivers' decision to engage with distractions it is the strategic (choosing when to engage) and decision (deciding to go through with it) stage that are of particular interest.

Horrey and Lesch (2009) explored the drivers' decision to engage with driver-initiated secondary tasks while driving on a test-track, an environment that offers increased levels of ecological validity than traditional simulators. They aimed to determine if drivers strategically adapt their engagement with distractions in line with the driving conditions but, in contrast to other findings (Lerner 2005; Metz et al. 2011), found no evidence to suggest that they did. Rather than strategically planning their interactions with the secondary task, Horrey and Lesch (2009) claim drivers chose to engage with the task independently of the road environment or task type and then breakdown the task into chunks to integrate the secondary task and primary task. This supported previous findings that drivers adapt their interaction at the control level (Haigney et al. 2000; Strayer et al. 2003), but not the strategic level. Yet, there were some aspects of the study that require further assessment, for example, the use of a test-track environment and lack of real-world motivations were cited as possible reasons for the lack of evidence for strategic planning (Horrey and Lesch 2009). Although the driving task is more realistic when driving on a test track in contrast to a driving simulator, drivers are still not exposed to 'true risk' of driving on the roads or interacting with other road users that are likely to influence their decision to engage. Some naturalistic driving studies have sought to explore the drivers' decision to engage with technology while driving using video recordings (Metz et al. 2015; Tivesten and Dozza 2015). They have demonstrated that the drivers' engagement with distractions is self-regulated and that drivers are able to strategically engage with tasks in line with predictable demands in the environment such as sharp bends, but that these strategies are ineffective when faced with unpredictable events such as a lead vehicle braking (Tivesten and Dozza 2015). Such naturalistic observation studies are, however, limited by their inability to understand why drivers engage with distractions as they only capture what drivers do (Tivesten and Dozza 2015). Further research is required to assess the drivers' decision to engage with distracting tasks within naturalistic conditions, as well as how this may vary to the use of driving simulators, which are a useful and commonplace tool in the assessment of driver distraction.

\subsection{The current study}

This study aimed to assess the drivers' decision to engage with technological devices while driving in a simulator and on the road in an instrumented vehicle (IV). Scenarios where drivers may need to interact with technology were posed to participants, while they drove along a pre-determined route. The drivers' decision was assessed at set points along the route through verbal protocol responses to the scenarios posed. The verbal protocols informed on the drivers' intention and, importantly, their decision-making process. The drivers' decision to engage and the contents of the verbal protocols were compared across the simulator and road condition. The potential for this method to be used to assess distraction without adversely effecting driving performance was also explored. This required the assessment of the drivers' average speed and speed variability when they provided verbal reports on their intention, compared to when they were driving without verbalising.

\section{Method}

\subsection{Participants}

Twelve participants (six male and six female) were recruited to take part in the study via advertisement at a University. Demographic information is presented in Table 2. All participants were required to hold a full driving license with 
Table 2 Demographics table

\begin{tabular}{llll}
\hline Demographics & Mean & SD & Range \\
\hline Age (years) & 39.75 & 11.8 & $26-58$ \\
Annual mileage (miles) & 10,733 & 4530 & $6000-21,000$ \\
Age of main vehicle (years) & 5.8 & 3.1 & $1-12$ \\
$\begin{array}{l}\text { No. years since passing driving test } \\
\quad \text { years) }\end{array}$ & 18.5 & 12.2 & $7-40$ \\
$\begin{array}{l}\text { No. of hours spent driving weekly } \\
\text { (hours) }\end{array}$ & 8.25 & 5.7 & $4-25$ \\
\hline
\end{tabular}

no more than three penalty points and to be over the age of 25 . This was required for the university insurance policy for on the instrumented vehicle (IV). Participants were also required to be frequent drivers, driving a minimum of twice weekly, and live local to Southampton to be familiar with the roads of the test route. Ethical approval was granted from the Universities Ethical Research Governance Office (ERGO 26046).

\subsection{Experimental design}

A repeated measures design was used with two driving conditions (simulator and IV). It assessed the drivers' decision to engage with a task in four different scenarios (read a text, change a destination, make a phone call, change a song) across three road types (Motorway, A road and roundabouts). This provided a methodology, whereby the question was embedded within scenarios to increase realism (Sharples et al. 2016). The decision to engage (yes or no) was measured through verbal protocols that included the drivers stated intention to engage with the task and their reasoning why. Voice recordings were transcribed and coded. Driving speed was also measured in both conditions. All scenarios were posed to participants across all three road types, with measures taken to ensure they were posed at the same points along the route when they drove on the road and in the simulator for all participants. The order of the simulator and road conditions were counterbalanced, as were the order of the scenarios across the road types.

\subsection{Equipment}

\subsubsection{Vehicles}

The vehicle used in the on-road trail was a Fiat Stilo (Fig. 1, left), an IV owned by the University. It was a right-hand drive with automatic transmission. For the simulator trial, the Southampton University Driving Simulator (SUDS) was used (Fig. 1, right). SUDS is a fixed base, right-hand drive Land Rover Discovery with automatic transmission. The simulator environment was created with STISIM M500W wide-field-of-view and was projected onto three screens in front of the vehicle and one behind, to give the rear-view. The simulator has a 135-degree field of view. Advanced driver-assistance systems (ADAS) were not used in either of the vehicles as the participants were required to manually drive the vehicle throughout the trials.

\subsection{Procedure}

The study took approximately $2 \mathrm{~h}$ to complete and was comprised of multiple stages that are summarised in Table 3. Participants were given an information sheet on arrival before being asked to give signed consent to participate. They were guided through the stages in Table 3 by the primary researcher until the end of the experiment, where they were reimbursed $£ 10$ for their time.

\subsubsection{Verbal protocol methodology}

Verbal protocol is a method that has become increasingly prevalent in driving behaviour studies (e.g., Young et al.
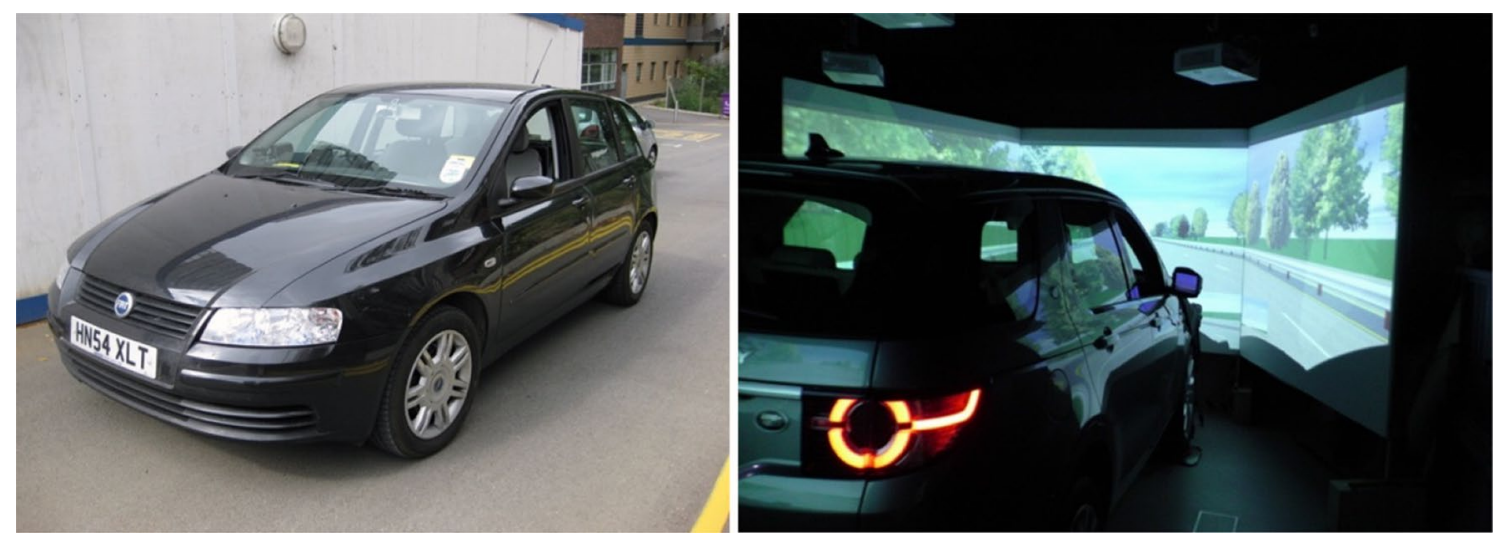

Fig. 1 Image of the IV used in the road condition (left) and simulator condition (right) 
Table 3 Breakdown on the study procedure into its comprising stages

\begin{tabular}{|l|l|c|}
\hline \multicolumn{1}{|l|}{ Study stages } & Approximate Time \\
\hline & Introductory briefing & $5 \mathrm{mins}$ \\
\hline & Training in verbal protocol (including a video session) & $10 \mathrm{mins}$ \\
\hline & Nre-trial interview to discuss scenarios & 10mins \\
\hline & Drive 2: Simulator/Road (including practise session) & 40mins \\
\hline & PASA TLX questionnaire & 40mins \\
\hline
\end{tabular}

2013; Banks and Stanton 2015; Salmon et al. 2017). The method aims to capture the contents of an individuals' working memory through their verbal reports in relation to the context and decisions that they are making (Ericsson and Simon 1993). It essentially requires participants to "think aloud' and generate a trace of their cognitive processing and the sequence of its unfolding from the point of view of the individual. The cognitive events can be traced from the point at which new information is introduced to a setting and the decision outcome that is then achieved. It is, therefore, a useful tool in assessing naturalistic decision making (Isenberg 1986; Gordon and Gill 1997). In this study, participants were required to produce verbal protocols of their decision-making process when posed within the task scenarios that would require them to interact with technology while driving. Importantly, drivers were not required to actually complete the tasks, they were just asked to verbalise their intention to engage given the current status of the road environment. This aimed to capture the participants' perceptions of the environment and how it would influence their strategies to engage with the technologies while driving.

Drivers were given training in how to perform the verbal protocol in stages. First, they were given a written overview of the verbal protocol procedure and what it was aiming to uncover, i.e., thinking aloud to produce a continuous stream of the drivers working memory (Ericsson and Simon 1993). The procedure, and what was expected of them during the study, was then further explained by the researcher. They were then shown a video of verbal protocol experts, which showed them participating in the study in the road condition, responding to the same scenarios in the same conditions that the participants would be asked. Participants were encouraged to ask questions at any point. The final stage allowed the participants to practise producing their verbal reports while driving during the practise phase of the route (see Sect. 2.4.3 for further details of the route). Both the simulator and road condition had a practise section that not only allowed the participant to acclimatise to the vehicle but also to practise responding to the scenarios that the researcher posed to them. This practise section lasted approximately $10 \mathrm{~min}$. The researcher gave feedback to the participant during this practise stage until they were confident that they were proficient. During the experimental trials, the researcher in the vehicle prompted the participant for further information where necessary during their verbal reporting. Participants were required to wear 
a head-mounted microphone that enabled high quality voice recordings that could be picked up over the noise of the vehicle while driving.

\subsubsection{Task scenarios}

To provide drivers with a consistent motivation for engaging with technological devices, pre-determined scenarios that would require technological engagement were developed by the researchers. These built upon the findings from the interview study by Parnell et al. (2017a) that probed drivers on a total of 22 different technological tasks that were drawn from the current literature investigating distraction from invehicle technology (e.g., Young and Lenné 2010; Neale et al. 2005; McEvoy et al. 2006; Harvey et al. 2011), as well as reports from road safety organisations and police reports (RAC 2016; Department for Transport 2015). The following tasks were selected from those studied by Parnell et al. (2017a) for further assessment in the current study: read a text, change a song, make a phone call and change a destination on a sat-nav. Scenarios were developed based on the motivations that participants of the interview study stated led them to engage with the technological device. They also relate to technologies which are commonly discussed in the literature and in the media (e.g., Kircher et al. 2004; Harvey and Stanton 2013; Lansdown 2012; Parnell et al. 2017b).

Participants were first presented with the scenarios in Table 4 during the introductory briefing. They were interviewed by the primary researcher on how they thought they would typically respond in these scenarios. This included asking if they had ever come across the scenario before, if they have the technologies mentioned available to them in their own vehicle and if so how do they typically interact with them. The interview was recorded on an Olympus digital voice recorder. These interviews enabled the researchers to establish the drivers' initial response to the scenarios and also how they would typically go about making the interactions with the technology, e.g., do they use voice-assisted commands, steering wheel buttons or hand-free phones.
These could then be referenced by the researcher during the trials for clarity if necessary. It was also useful to establish the different ways in which the four common scenarios could be responded to.

\subsubsection{Route}

The same pre-determined route was driven in both the simulator and the road condition. The study was conducted between 10.30 am and $3 \mathrm{pm}$ on weekdays only to ensure that the conditions on the road avoided periods of heavy traffic (i.e., rush hour). The study was also only conducted in dry weather conditions. The route was chosen as it started and ended at the University and covered the different road types of interest (A road, motorway and roundabouts). It was 13.2 miles long taking approximately $40 \mathrm{~min}$ to drive (including the practise segment). The simulated route was designed to replicate the real-world as much as possible using the STISIM software. This included road curvature, traffic density and the development of salient localised objects with the creation of a custom library of models that aimed to replicate objects on the real-world route. For further information on this route, as well as the pilot study, see Allison et al. (2017).

Figure 2 shows a map of the route that the participants were given during the introductory briefing. Participants were encouraged to understand, where the route would take them, but they did not need to memorise it as the primary researcher sat in the passenger seat of the car in both the simulator and road condition to provide directions. The dotted black line represents the practise segment and the bold grey line the test segment. The arrows show the direction that the participants drove the route. The structure of the study meant that participants were asked to state their intention to respond to the technologies in each of the four scenarios on each of the three road types; motorway, A road and roundabouts. This required participants to produce 12 separate verbal protocol reports. The key in Fig. 2 shows, where the researcher asked the participants their intention to engage with the technology in the scenarios. The same

Table 4 Scenarios relating to different technology use

\begin{tabular}{ll}
\hline Task & Scenario \\
\hline Read text on a mobile phone & $\begin{array}{c}\text { You receive a text message which sets off the alert tone and causes the phone to light up. Being aware that you } \\
\text { have received a text message. Would you read the text at this moment in time? }\end{array}$ \\
Change destination on a sat-nav & $\begin{array}{r}\text { You are driving on a long journey to a destination you are not familiar with and, therefore, are using a sat-nav } \\
\text { to direct you. Along the way, you realise that you need to stop off at an alternative destination that is not cur- } \\
\text { rently programmed into your sat-nav. Would you enter the new destination into the sat-nav? } \\
\text { You are driving to a meeting but are running very late due to heavy congestion and you will not be able to } \\
\text { reach them at the time you prearranged. Would you call the person you are due to meet to let them know you } \\
\text { are running late? } \\
\text { You are driving while listening to the radio when a song comes on the radio that you really do not enjoy listen- } \\
\text { ing to. Would you change the radio station to find one that is playing music that you do like? }\end{array}$ \\
Change the song/radio station &
\end{tabular}




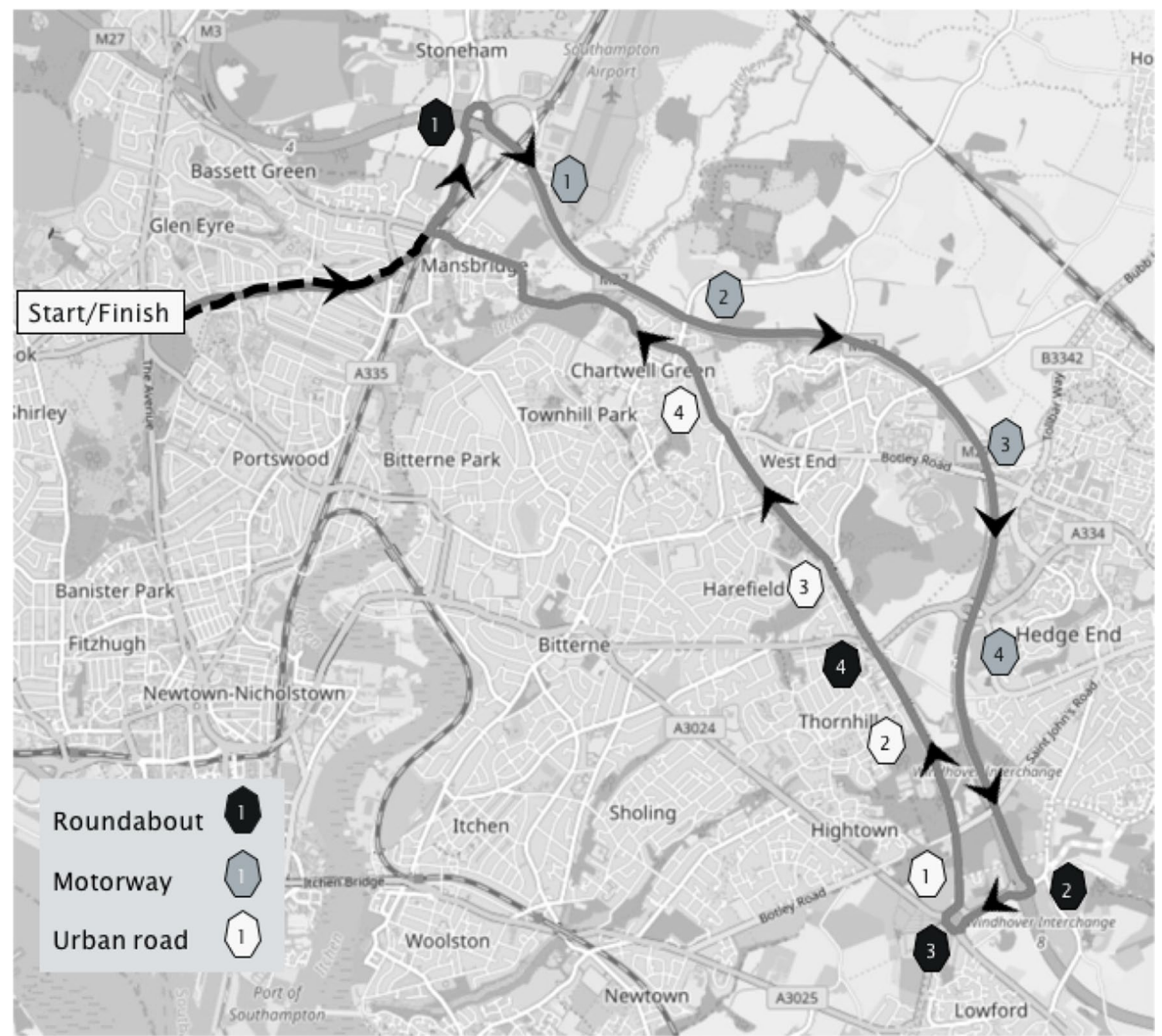

Fig. 2 Map of the route with directional arrows and the location the scenarios were posed

primary researcher conducted all the trials for consistency, ensuring that they posed the scenarios at the same points across all participants. Verbal reports lasted approximately $60 \mathrm{~s}$ seconds each time. The order in which the scenarios were asked were randomised between participants as was the order of the simulator and road trial. Yet, within participants, the scenario order was the same in the road and simulator condition. The researcher made sure that they were always fully alert and aware of the road environment and so only interacted with the participant at times that they felt it was safe to do so. The participant was also instructed to respond when they felt able to.

\subsubsection{Workload}

The NASA-TLX questionnaire (Hart and Staveland 1988) was administered to participants after the road trial and the simulator trial to assess their reported workload when they were asked to respond to the scenarios. The subjective workload of the participants when providing verbal responses in the simulator could then be compared to the road.

\subsection{Data analysis}

Qualitative data were obtained by transcribing the recordings from the participants' verbal reports; these were then input into Nvivo11 for further coding and analysis. The drivers' intention to engage was coded as 'yes' or 'no'. Their verbalisations of their interpretation of the current road environment and how this influenced their intention to engage were coded with the thematic framework developed by Parnell et al. (2017a), see Table 1. The use of Nvivo11 to code the qualitative data also facilitated quantitative analysis to be run on the frequency of references made to the nodes of interest. To determine if responses in the simulator were consistent with responses on the road, Matthews (1975) correlation 
coefficient (Phi) was calculated. Phi is typically used in machine learning as it calculates the correlation between the predicted and observed binary outcomes. The measure has been used to assess the validity of a range of popular Human Factors methods (Stanton and Young 1999). It was applied here to determine the correlation between the frequency of yes/no responses reported in the simulator and the road condition. An interaction between the response given, the road type and task type was explored using a three-way loglinear analysis. The analysis aimed to identify if drivers were more likely to respond one way or another on specific road types or in relation to different tasks, as well as assessing any interacting effects between these factors.

The number of references made to the thematic framework (Parnell et al. 2017a; Table 1) were also correlated between the road and the simulator condition to assess the relationship between what drivers rate as important to their decision-making process when engaging with technology on the road and in the simulator. A Spearman's rank correlation was conducted to correlate the frequency of responses to each of the themes across both conditions.

Quantitative data were also obtained from other metrics, including the drivers' workload, average speed and speed variability while driving, for statistical analysis. Paired samples $t$ tests were run on the drivers rated workload in the simulator and on the road. Measures of the drivers' average speed and variation in speed when driving with and without providing verbal protocols were obtained from the simulator and road condition. Speed metrics could only be obtained and compared on the motorway and A road, but not on the roundabouts as breaking inputs could not be generated from the IV. The motorway and A road were split into segments, where the drivers were verbalising their intention and where they were driving without verbalising their intention. Periods of comparable speed limit and road curvature were compared across the route for the analysis of speed when they were providing verbal reports and when they were not. Areas where external sources may have influenced the drivers speed were removed such as intersections on the A road and junction exits on the motorway, as well as $100 \mathrm{~m}$ before and after them (Salmon et al. 2017). The speed on the motorway and A road were compared separately to assess if there were any differences in the metrics between these road types. Comparisons between the road and the simulator could not be conducted due to the different sampling rates with which the speed data were obtained during these trails. Speed was not found to be normally distributed in the simulator condition; therefore, Wilcoxon signed rank tests were performed to assess the effect of performing verbal protocols on the A road and the motorway. On the road, paired sample $t$ tests were used to assess average speed on the A road and motorway. Again, speed variability in the simulator was assessed with a non-parametric Wilcoxon signed rank test. Whereas paired sample $t$ tests were run on the road data to compare the effect of verbal protocol on speed variability when driving on A roads and motorways.

\section{Results}

The study aimed to assess the drivers' intention to engage with in-vehicle technologies in a variety of different scenarios while driving in the simulator and on the road. Assessment of the drivers' metrics also allowed the assessment of how providing verbal protocols in response to the scenarios impacted on the drivers' speed.

\subsection{Scenario responses}

\subsubsection{Pre-trial interview}

The pre-trail interview assessed the different ways that the participants stated they would interact with the tasks presented to them in the scenarios. These are shown in Table 5.

\subsubsection{Stated intention}

Intention to engage with technologies in the simulator environment was compared to intention in the real-world using Matthews (1975) correlation coefficient (Phi). The frequency of yes/no responses in the simulator and on the road were calculated and classified as follows (Table 6):

Hit: Simulator = 'yes' and Road = 'yes'.

Miss: Simulator $=$ 'no' and Road $=$ 'yes'.

False Alarm (FA): Simulator $=$ 'yes' and Road = 'no'.

Correct Rejection (CR): Simulator $=$ 'no' and Road $=$ 'no'.

Calculation of Matthews (1975) correlation coefficient returned a phi value of 0.68 . This suggests a strong positive

Table 5 Different ways that participants stated they would interact with the tasks in the scenarios posed in the pre-trial interview

\begin{tabular}{ll}
\hline Task scenario & Type of interaction \\
\hline Read text on mobile phone & $\begin{array}{l}\text { Read who it was from } \\
\text { Glance at the first line } \\
\text { Open the text } \\
\text { Enter pin code to read } \\
\text { Recent destination }\end{array}$ \\
Change destination on a sat-nav & Enter post code manually \\
& Voice command \\
Vake a phone call & Via the phone \\
& Use voice command to initiate \\
Change the song/radio station & Steering wheel button \\
& Central console button \\
& Phone
\end{tabular}


Table $62 \times 2$ Contingency table of responses on the road and in the simulator

\begin{tabular}{lll}
\hline & \multicolumn{2}{l}{ Road } \\
\cline { 2 - 3 } & Yes & No \\
\hline Simulator & & \\
Yes & (Hit) 77 & (FA) 5 \\
No & (Miss) 18 & (CR) 44 \\
\hline
\end{tabular}

Table 7 Frequency of 'yes' and 'no' responses across road and task type

\begin{tabular}{llrr}
\hline Road type & Scenario & \multicolumn{2}{c}{$\begin{array}{l}\text { Frequency of } \\
\text { responses }\end{array}$} \\
\cline { 3 - 4 } & & Yes & No \\
\hline A road & Phone & 15 & 9 \\
& Text & 16 & 8 \\
& Destination & 14 & 10 \\
Motorway & Song & 22 & 2 \\
& Phone & 16 & 8 \\
& Text & 17 & 7 \\
\multirow{2}{*}{ Roundabout } & Destination & 16 & 8 \\
& Song & 21 & 3 \\
& Phone & 9 & 15 \\
& Text & 10 & 14 \\
Total & Destination & 7 & 17 \\
\hline
\end{tabular}

correlation between drivers reported intention to engage with the technology in the simulator and on the road.

\subsubsection{Road type and task type}

As the frequency of responses across the simulator and the road were found to have a strong positive correlation, the frequency of 'yes' and 'no' responses were aggregated across both the road and simulator conditions. The frequency of responses is shown in Table 7.

A three-way loglinear analysis produced a final model that retained two-way effects. The highest order interaction (task $\times$ road $\times$ responses) was not found to be significant $\left[X^{2}(6)=1.49, p=0.96\right]$. The level two interaction was significant $\left[X^{2}(17)=41.218, p<0.01\right]$. To break down this effect, separate Chi square tests were run for responses to task type and road type. For task type, there was a significant association between task type and responses (yes or no) $\left[X^{2}(3)=13.77, p=0.03\right]$. Odds ratios suggested that participants were 2.40 times more likely to change a song than make a phone call, 2.47 times more likely to change a song than reading a text and 3.60 times more likely to change a song than changing a destination on a sat-nav. The direction of these differences can be seen in Fig. 3a. For road type, there was a significant association between road type and response $X^{2}(2)=24.01, p<0.01$. The odds of drivers responding yes to engaging with a task on a roundabout was 3.77 times lower than on the motorway and 3.24 times lower than on an A road, (see Fig. 3b).

\subsubsection{Reasons for stated intention}

While the drivers' stated intention was strongly correlated in the simulator road conditions, it was also of interest to assess if the drivers gave the similar reasoning for their intentions across both research settings. The frequency of references that were coded to each of the themes from the previously generated thematic framework (Parnell et al. 2017a, Table 1) are shown in Table 8.

Table 8 shows that a theme has been added to the original framework that is presented in Table 8; 'Other road users'. The coding procedure revealed that there were a frequent number of meaningful references to other road users that influenced the drivers' intentions to engage with other cars, lorries, cyclists and pedestrians. Therefore, a new theme and its corresponding subthemes were added to the framework to capture these references. The coding of the themes followed the same procedure as that used in the development of the original framework (Parnell et al. 2017a). The descriptive themes (e.g., behind, in-front, oncoming etc.) are shown in Table 1 to indicate what the subthemes are referencing. Analysis of the remainder of the results will focus on the
Fig. 3 Frequency of responses across a road type and $\mathbf{b}$ scenario type (a)

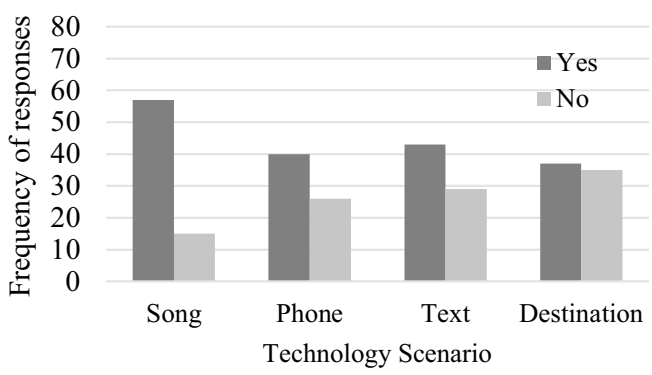

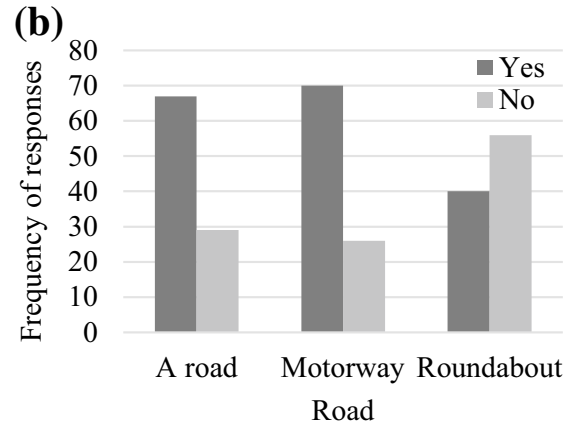


Table 8 Frequency of references coded to the thematic framework developed by Parnell et al (2017a) in the road and simulator conditions

\begin{tabular}{|c|c|c|}
\hline Key Themes & Road & Simulator \\
\hline Context & 42 & 33 \\
\hline Journey & 12 & 11 \\
\hline Road & 8 & 2 \\
\hline Task & 22 & 20 \\
\hline Driver & 33 & 34 \\
\hline Attitude of the driver & 30 & 26 \\
\hline Influence of others & 1 & 2 \\
\hline Tendency & 1 & 0 \\
\hline View of self & 1 & 6 \\
\hline Infrastructure & 247 & 224 \\
\hline Illegality & 0 & 2 \\
\hline Perception of surrounding environment & 61 & 53 \\
\hline Road Layout & 35 & 33 \\
\hline Road-related behaviour & 73 & 65 \\
\hline Task-road Relationship & 78 & 71 \\
\hline Task & 137 & 125 \\
\hline Ability to complete & 5 & 3 \\
\hline Complexity & 29 & 16 \\
\hline Desirability & 1 & 4 \\
\hline Duration & 24 & 14 \\
\hline Engagement Regulation & 28 & 33 \\
\hline Interaction & 50 & 55 \\
\hline Other road users & 177 & 165 \\
\hline Car & 76 & 63 \\
\hline Behind & 23 & 10 \\
\hline In front & 22 & 20 \\
\hline On coming & 3 & 7 \\
\hline Overtaking & 1 & 5 \\
\hline Parked & 2 & 4 \\
\hline Predictability & 9 & 12 \\
\hline Speed & 5 & 3 \\
\hline Turning & 5 & 4 \\
\hline Cyclist & 2 & 0 \\
\hline Lorry & 8 & 9 \\
\hline overtaking & 8 & 9 \\
\hline Pedestrian & 11 & 17 \\
\hline children & 2 & 2 \\
\hline
\end{tabular}

Grey text indicate new themes semantic subthemes only, in line with the original themes from the framework.

It can be seen from Table 8 that the number of references to each of the key themes in the road condition was similar to the number of references in the simulator condition. A Spearman's rank correlation coefficient showed a very strong positive correlation between the frequency of references to the systems and sematic themes given for engaging or not engaging with the technology in the simulator and on the road, $r(27)=0.96, p<0.01$. This suggests that, not only were the responses similar in the simulator and on the road, but the reasons that drivers gave for their response were highly correlated as well.

\subsubsection{Matrix queries}

The loglinear analysis suggested that drivers were less willing to engage with the tasks on roundabouts in contrast to the motorway and A road. They were also more likely to respond to the song change scenario than the other technological 
scenarios. The coded transcripts were analysed to explore why this may be the case. A matrix coding query was run in Nvivo11 to revel the frequency of references made to each of the themes in the framework across each of the tasks (Table 9) and each of the road types (Table 10).

Table 9 shows that the song scenario had fewer references to the infrastructure themes than the other scenarios and more references to the task themes. The task references that were made in response to the song scenario reflected a unanimous agreement on the ease of completing the task which increased their propensity to engage with it across road types.

"I'd probably glance once and then I would just know what I' $m$ doing. I can feel the buttons so I'd be quiteI'd have no problem doing that" (Participant 1).

"That again is because it is a quick action to do in my car" (Participant 2).

Table 9 Frequency of references to the themes in the framework across each of the scenarios

\begin{tabular}{|c|c|c|c|c|}
\hline Themes & Destination & Phone & Song & Text \\
\hline Context & 21 & 21 & 9 & 24 \\
\hline Journey & 7 & 7 & 3 & 6 \\
\hline Road & 3 & 1 & 3 & 3 \\
\hline Task & 11 & 13 & 3 & 15 \\
\hline Driver & 11 & 18 & 13 & 25 \\
\hline Attitude of the driver & 8 & 12 & 12 & 24 \\
\hline Influence of others & 2 & 0 & 0 & 1 \\
\hline Tendency & 1 & 0 & 0 & 0 \\
\hline View of self & 0 & 6 & 1 & 0 \\
\hline Infrastructure & 121 & 108 & 86 & 104 \\
\hline Illegality & 1 & 1 & 0 & 0 \\
\hline Perception of surrounding env & 24 & 32 & 27 & 31 \\
\hline Road Layout & 12 & 18 & 18 & 20 \\
\hline Road-related behaviour & 40 & 37 & 23 & 38 \\
\hline Task-road relationship & 56 & 37 & 26 & 30 \\
\hline Task & 42 & 60 & 69 & 55 \\
\hline Ability to complete & 1 & 3 & 1 & 3 \\
\hline Complexity & 12 & 15 & 13 & 5 \\
\hline Desirability & 2 & 0 & 3 & 0 \\
\hline Duration & 10 & 7 & 15 & 6 \\
\hline Engagement regulation & 6 & 11 & 8 & 36 \\
\hline Interaction & 16 & 36 & 42 & 11 \\
\hline Other road users & 28 & 31 & 30 & 36 \\
\hline Car & 29 & 32 & 35 & 38 \\
\hline Cyclist & 0 & 0 & 1 & 1 \\
\hline Lorry & 4 & 3 & 4 & 6 \\
\hline Pedestrians & 5 & 11 & 5 & 7 \\
\hline
\end{tabular}

Table 10 Frequency of references to the themes in the framework across each of road types

\begin{tabular}{lrrr}
\hline Key themes & A road & Motorway & Roundabout \\
\hline Context & 28 & 20 & 27 \\
Journey & 4 & 4 & 15 \\
Road & 5 & 4 & 1 \\
Task & 19 & 12 & 11 \\
Driver & 17 & 25 & 25 \\
Attitude of the driver & 11 & 24 & 21 \\
Influence of others & 2 & 0 & 1 \\
Tendency & 0 & 1 & 0 \\
View of self & 4 & 0 & 3 \\
Infrastructure & 120 & 123 & 176 \\
Illegality & 1 & 1 & 0 \\
Perception of surrounding env & 37 & 41 & 36 \\
Road Layout & 36 & 16 & 16 \\
Road-related behaviour & 38 & 33 & 67 \\
Task-road relationship & 21 & 47 & 81 \\
Task & 85 & 86 & 55 \\
Ability to complete & 1 & 4 & 3 \\
Complexity & 18 & 20 & 7 \\
Desirability & 3 & 2 & 0 \\
Duration & 15 & 10 & 13 \\
Engagement regulation & 21 & 23 & 17 \\
Interaction & 38 & 44 & 23 \\
Other road users & 200 & 80 & 42 \\
Car & 2 & 36 & 22 \\
Cyclist & 3 & 13 & 0 \\
Lorry & 26 & 1 & 1 \\
Pedestrians & & & 1 \\
\hline & 36 & \\
\hline
\end{tabular}

There was less focus on the road type and the infrastructure for the song task as drivers claimed it did not influence their decision.

"Even when diving across lanes, probably could change a song quite quickly" (Participant 3 ).

"Yeah, I would change that now, straight away. I probably wouldn't spend much time assessing the situation I would probably just reach out and change it" (Participant 5).

Whereas for the other tasks, drivers talked more about how they may (or may not) integrate their technological interactions with the technology with the current road environment, referencing the 'task-road relationship' theme.

"I would wait until I've kind of done... well, I'd either kind of slow down in this lane or I'd wait to do my manoeuvre" (Participant 2).

"I would do the voice entry, but I wouldn't want to take my hands off the wheel right now because it's quite a 
windy road and the guy in front of me is quite slightly erratic, so I'd try the voice thing but I wouldn't try to on a touch screen" (Participant 11).

There were proportionally more references to the 'engagement regulation' theme under the task category for the 'reading a text message' scenario. These references relate to statements suggesting they may initially be drawn to the text upon receiving it, and may read who it was from, before determining if they would read the whole text. They suggest how the presentation of the text would initially guide their attention to it, and they would then assess how they would then regulate their engagement with it.

“once I've had a look at who the text message was from, I would assess whether I wanted to look at it or not, depending on how important the person was to me" (Participant 3).

"I'd read like the first line of it, but I probably wouldn't open the text up to read the rest of it unless it was all shown on the front anyway" (Participant 11).

When assessing references to the themes by road type, Table 10 shows that the A road had a larger number of references to the 'other road users' theme than the motorway or the roundabout road type. When driving on this road, participants verbal reports were strongly influenced by the presence of others in the road environment including pedestrians as well as the unpredictable nature of the A road. This was in contrast to the motorway that was perceived as a more stable environment.

"Yeah, so there's no cars behind me, there's no pedestrians, it's quite quiet, there's a few cars coming towards me" (Participant 1).

"Yep so a massive gap to the car in front, there's absolutely nothing has followed me from the roundabout behind, so yeah I could flick through the radio stations to my heart's content" (Participant 3).

The roundabout road types, which had significantly fewer 'yes' responses, had more references to the infrastructure theme and less references to the task theme than the A road and motorway. The frequent references to the 'task-road' relationship, suggest that the attention required when navigating a roundabout limited their ability to engage with a secondary task.

"I'm waiting at the roundabout because there's a car in front of me which has just gone, so I'm looking at whether I can go. And I'm looking at the roundabout and getting onto the roundabout at the moment. No,
I've got to do too much manoeuvring of the car and thinking about the environment to bother looking at my phone" (Participant 1).

On the motorway, references to the infrastructure themes frequently mentioned the road environment and the 'roadrelated behaviour' theme, including the behavioural differences across the motorway lanes that influenced their willingness to engage.

"Probably there I would have delayed slightly until I was in the right lane, the left lane" (Participant 3).

"I'd stay in this lane. I wouldn't try and change lane while I did it, and I'd try and keep... leave like a good distance between me and whatever was in front and just try and keep an eye out for someone pulling into that gap while I was on the phone" (Participant 11). "Again, I'd pull into the slow lane, and then I'd probably do it now, yeah" (Participant 12).

\subsection{Driving speed}

Measures of the drivers' average speed and variation in speed when driving with and without providing verbal protocols on their intention to engage with technology were obtained for the simulator and the road condition. The speed on the motorway and A road were compared separately to assess if there were any differences in the metrics between these road types. Average speed and the standard deviation of speed are presented in the box plots in Figs. 4 and 5, respectively.

\subsubsection{Mean speed}

No differences were found between the drivers' mean speed in the simulator when they were providing verbal responses compared to when they were driving without verbally responding on either the A road, $[(Z=-0.55, p=0.58)$, $r=-0.11]$ or the motorway, $[(Z=-1.10, p=0.272)$, $r=-0.22]$. There were also no significant differences in mean speed when verbalising responses compared to driving without verbalising responses on the motorway in the onroad condition, $[t(11)=-0.16, p=0.876, r=0.89]$. There was, however, a significant difference on the A road when drivers were driving on the road, with an increased average speed when driving without providing verbal reports $(\mathrm{M}=35.46, \mathrm{SD}=2.74)$, compared to when they were providing verbal responses $(\mathrm{M}=32.39 \mathrm{mph}, \mathrm{SD}=1.87)$, $[t(11)=-4.77, p<0.01, r=0.44]$. 
Fig. 4 Box plot to show the differences in the average speed across the motorway and A road when driving with and without VP. The road condition and simulator condition are presented

Fig. 5 Box plot to show the differences in the standard deviation of speed across the motorway and A road when driving with and without VP.

The road condition and simulator condition are presented

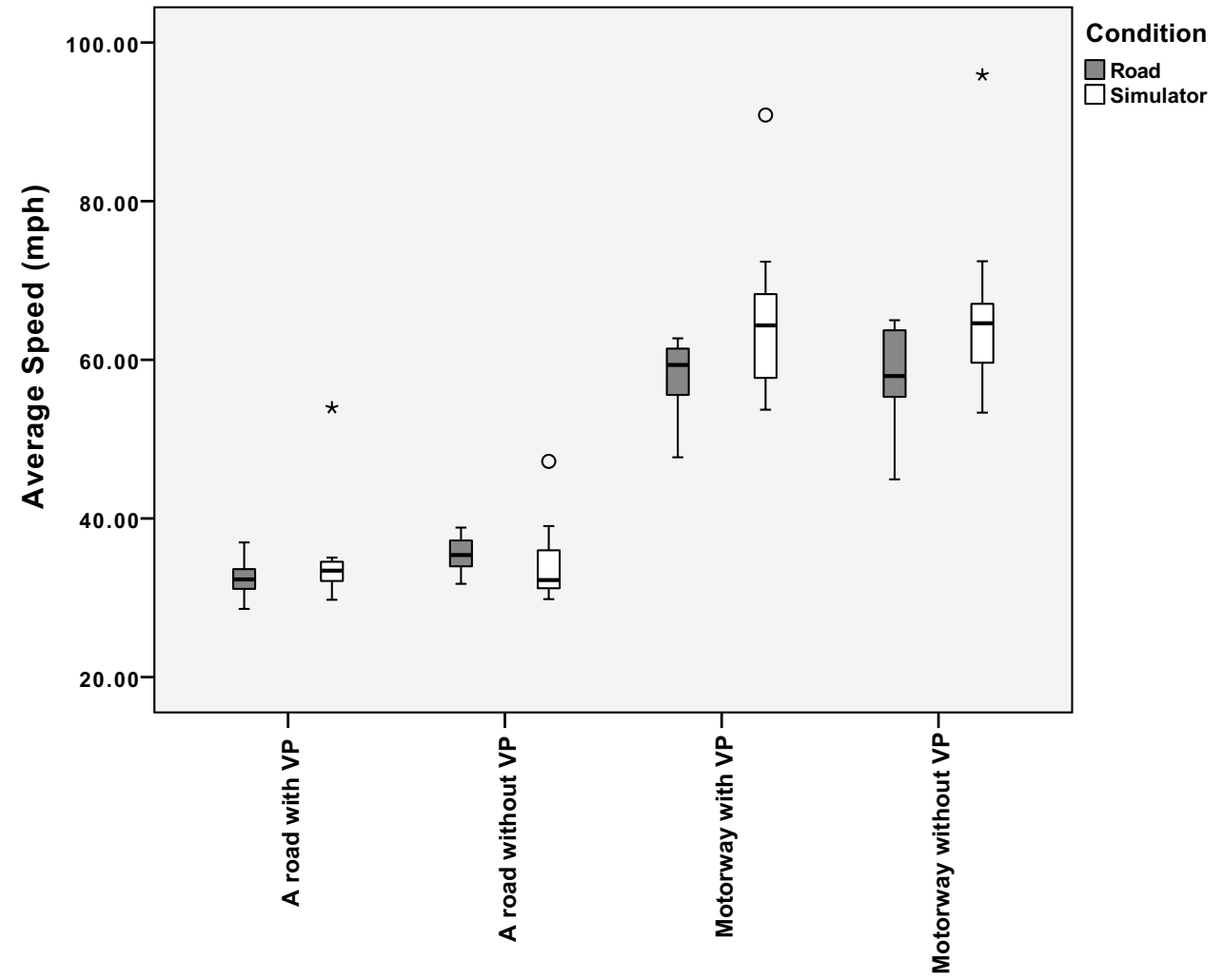

Road type and Verbal Protocol (VP) condition

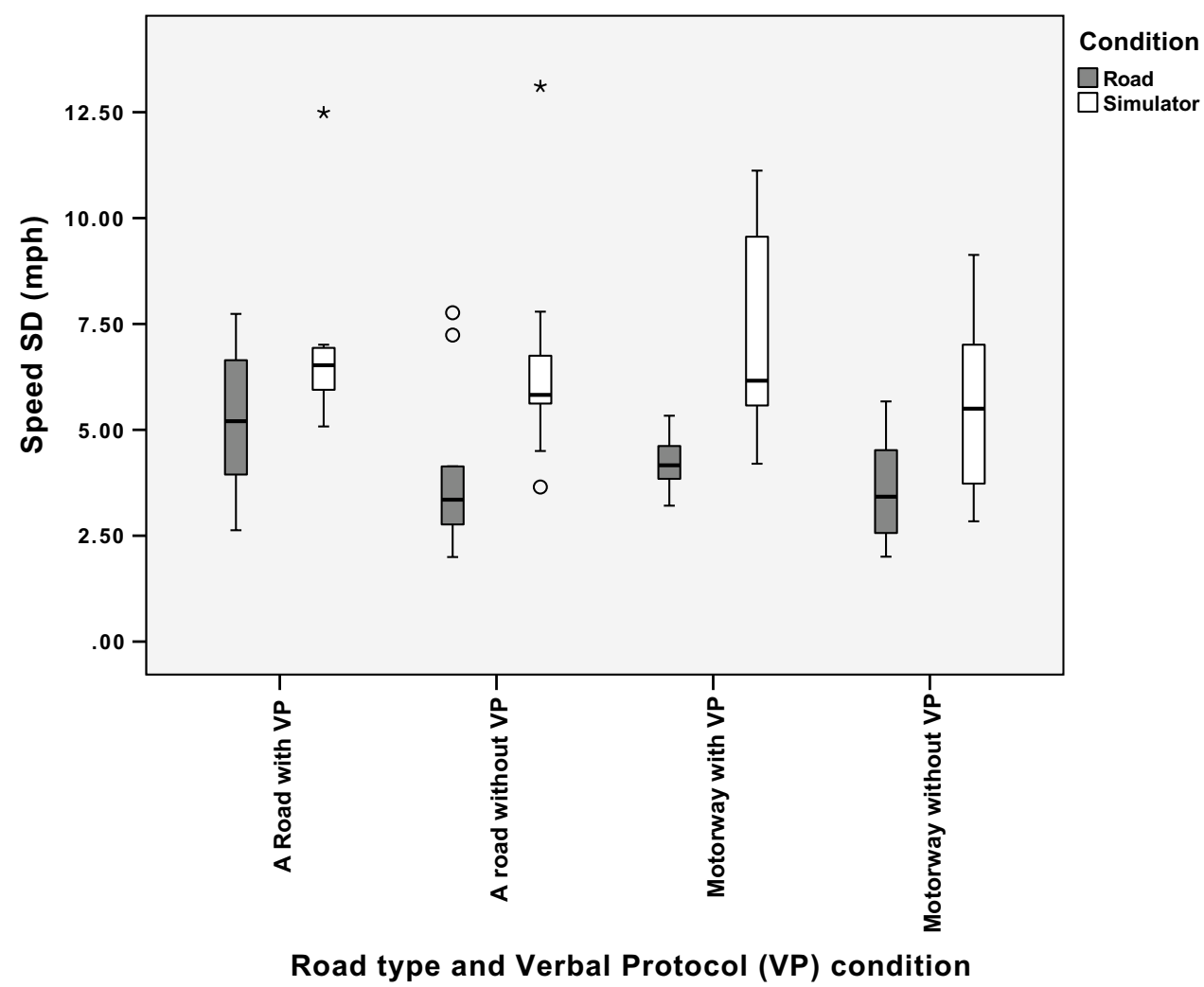


Fig. 6 Boxplot showing the workload scores for the simulator and road condition

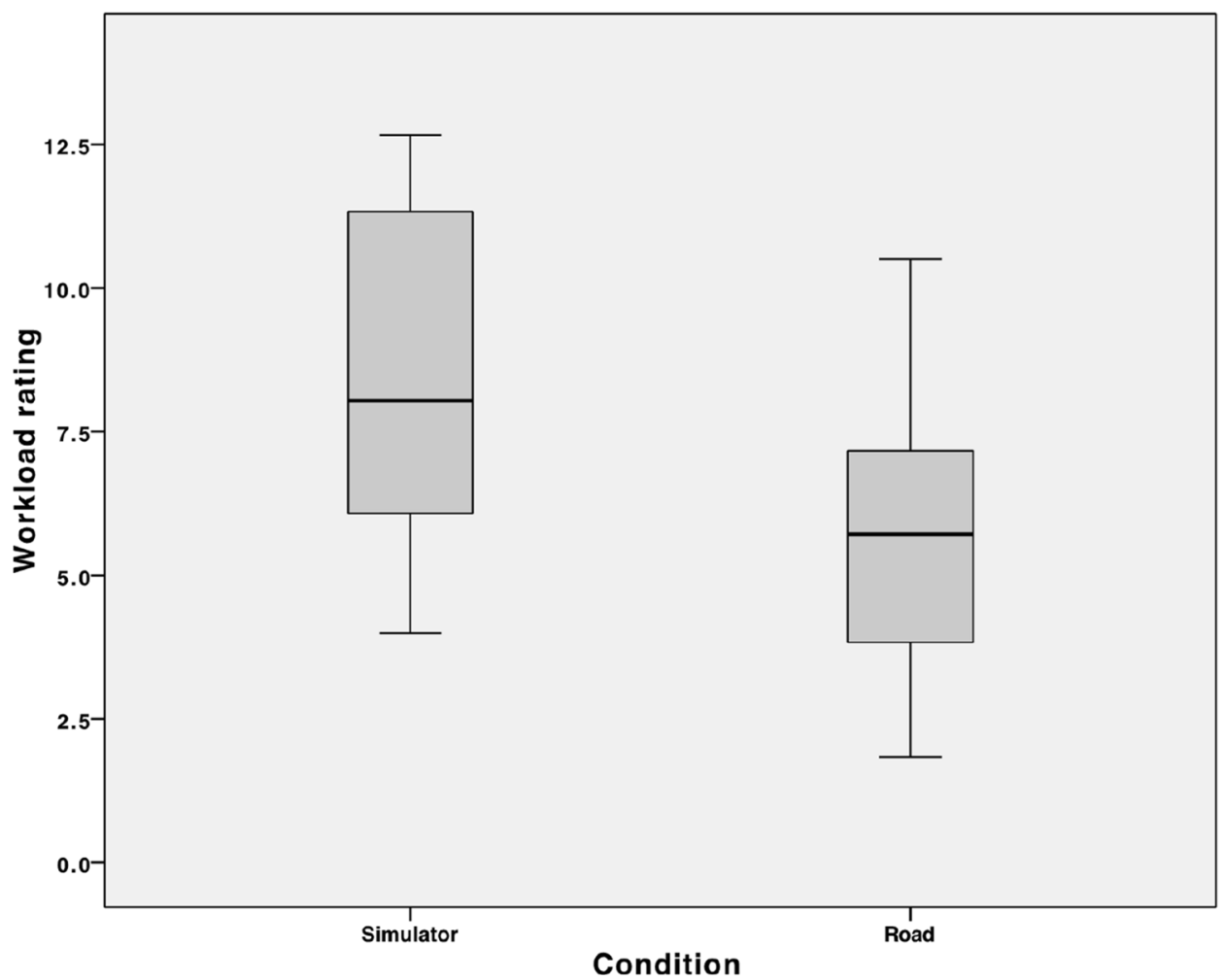

\subsubsection{Speed variability}

In the simulator condition, there was no significant difference in speed variability on the A road when providing VP compared to when they were not, $(Z=-1.26, p=0.21$, $r=-0.26$ ). Yet, there was a significant difference when they were driving on the motorway in the simulator, with increased variability when providing verbal responses $(\mathrm{M}=7.28, \mathrm{SD}=2.33)$ compared to driving without verbal responses $(\mathrm{M}=5.55, \mathrm{SD}=1.99),(Z=-2.67, p<0.01$, $r=-0.54)$. In the on-road condition there was no significant difference in speed variability on the motorway, [ $t=-1.198, p=0.081, r=-0.38]$, or the A road (after correcting for the multiple test with a Bonferroni correction), $[Z=-2.20, p=0.028, r=-0.45]$.

\subsection{Workload}

An overall workload score was obtained for the simulator and road condition by averaging the six subscales of the measure (mental, physical, temporal, performance, effort, frustration) (e.g., Horberry et al. 2006). Workload ratings were found to be significantly higher in the simulator condition $(\mathrm{M}=8.41, \mathrm{SE}=0.87)$ than in the road condition $(\mathrm{M}=5.61, \mathrm{SE}=0.70),[Z=-2.82, p<0.01, r=-0.58]$

(Fig. 6).

\section{Discussion}

Previous research has suggested that drivers voluntarily engage with distractions (Beanland et al. 2013). While there is evidence to suggest that drivers may regulate their behaviour in a way that would encourage them to be distracted (Dogan et al. 2011; Horrey and Lesch 2009; Tractinsky et al. 2013), there is also a movement within the road transport domain to study how driver behaviour may be the result of interactions between multiple interdependent actors in the sociotechnical system (Tingvall et al. 2009; Larsson et al. 2010; Salmon et al. 2012; Lansdown et al. 2015; Parnell et al. 2016). This study has sought to understand the drivers' decision to engage with different technological tasks (change a song, make a phone call, read a text and change a destination) in predefined scenarios across different road environments (A road, motorway and at roundabouts) and between study environments (on the road verses in the simulator). Previous assessment of the drivers' decision to engage with secondary tasks, while driving has utilised interview studies (e.g., Parnell et al. 2017a), driving simulators (Metz et al. 2011; Schömig and Metz 2013), test tracks (Horrey and Lesch 2009) and naturalistic driving studies (Tivesten and Dozza 2015; Metz et al. 2015) with varied findings. While the high level of control obtained in simulator and test-track experiments allows for the manipulation of variables, they do not allow for the contextual factors that surround driving behaviour in the real world to be assessed. Meanwhile, 
naturalistic driving studies are able to capture the drivers' decision to engage relative to real-world driving conditions, yet they cannot obtain the underlying reasons why drivers choose to engage with the task. The interview study conducted by Parnell et al. (2017a), however, facilitated the varied causal factors that drivers state influences their decision to engage to be constructed into a thematic framework. This was applied in this study to the drivers' verbal reports on their intention to engage in the posed scenarios in the simulator and road environment. This provided a validation of the inductively generated thematic framework from the interview while also expanding it to account for an additional 'other road users' theme. The study of drivers' decisions when performing the act of driving resulted in numerous references to 'other road users' that were not captured in the interview study and thus further highlights the importance of assessing decisions within the context that they emerge. This compliments other decision-making studies that have found driver behaviour to be influenced by other drivers (Pedic and Ezrakhovich 1999). Yet, it diverges from the findings of the naturalistic study conducted by Tivesten and Dozza (2015) who observed that drivers' decisions to engage with visual-manual tasks was not influenced by the presence of lead vehicles. The drivers' verbal responses in this study suggests that drivers are actually very aware of the other vehicles on the road.

\subsection{Experimental condition}

A strong positive correlation was found between the drivers' decision to engage in secondary tasks, both in the simulator and on the road. Furthermore, the key themes that drivers referenced in their reasoning for their intention were also strongly correlated on the road and in the simulator. This provides validation to the simulation developed for the study, it also shows promise for the use of simulators to observe, measure and influence the drivers' intention to engage with technological sources of distraction for future research. Furthermore, the application of the inductively generated thematic analysis from an interview study (Parnell et al. 2017a), to the drivers' verbal reports when driving in a simulated and real-world environment suggests that participants are able to generate realistic decision-making processes in an interview setting.

\subsection{The effect of road type and task type on naturalistic decision making}

Analysis of drivers stated intention to engage across road types and task types showed evidence for strategically adapted engagement. This supports the findings of Metz et al. (2011) in the simulator and Tivesten and Dozza (2015) in their naturalistic driving study, but opposes the lack of strategical adaption found by Horrey and Lesch (2009) on the test-track. The frequency of themes that were coded to the drivers' verbal responses in Tables 9 and 10 highlighted how the themes may vary in their influence over the driver decision to engage across tasks and road types, respectively. Acknowledging that the drivers' decision to engage with possible distracting technologies is dependent on a large range of factors that are likely to vary across different scenarios is very important. It is key that road and vehicle designers understand how their choices may impact on the drivers' decision to engage with tasks that may lead them to become distracted. Tables 11 and 12 in the "Appendix" are intended to summarise to the key factors that influence the decision to engage across tasks and road types. These highlight both the factors that are likely to increase the drivers decision to engage and those that would decrease their likelihood of engaging. As the reports were not found to differ across the road and simulator conditions, these factors were drawn across experimental environments and, therefore, can also be applied to both environments. These tables may be on interest to road and vehicle designers to guide their own decisions on what facilities they provide the driver and how they can shape their behaviour. Further discussion around these points are presented below for both task type and road type.

\subsubsection{Task type}

Drivers were two to three times more likely to respond to the 'changing song' scenario than the scenarios requiring 'phone', 'text', or 'destination change' responses. This supports previous findings from studies, where drivers were asked to rank tasks on their potential for distraction, where in-car entertainment systems and radio controls are typically ranked lower than phone based or sat-nav interactions that are perceived to be more distracting (Young and Lenné 2010; Lansdown 2012). The verbal reports that were coded to the thematic framework suggest that drivers were less concerned with road type when deciding whether to engage with the song task, but were motivated by the ease of the task and the limited attention it took away from the driving task. This supports previous findings by Lerner (2005) in the real-world environment. Intention was lower for other tasks (phone, text and navigation), with issues frequently referencing the complexity the task. Yet, where drivers did state they would engage, they reported their behaviour would involve several steps that would lead them to approach the task in a staged manner while adapting their driving behaviour. For example, "if I had my sat-nav kind of up here I could start to slowly enter the destination and just kind of maybe doing one step at a time and then looking back to the road to make sure nothing's changed" (Participant 3 ). These findings suggest that, once drivers have made the strategic 
decision to engage with the task, they adapt their behaviour on a momentary basis to integrate the primary and secondary task, a feature of adaption at the control level (Schömig and Metz 2013). Harvey and Stanton (2013) found that drivers were able to use 'shared glances' to monitor both the road and a visual display at the same time once a task had been engaged with, to perform the two tasks concurrently. The use of shared glances was very apparent when entering destinations into a navigation display (Harvey and Stanton 2013). The behaviour drivers discussed in their verbal reports was suggestive of this 'shared glance' model; drivers are aware of their behaviour and can verbalise their strategies for adapting their attention to achieve their goals.

The scenario that asked drivers if they would read a text that they had just received, frequently referenced the 'engagement regulation theme' which captures references to how initiating a task is regulated. This is due to the number of participants who stated that they would read a text message if they were alerted to it first, to see who it was from and then assess if they wanted to go on to read the text in full. Others claimed their device would allow them to read the first few lines without touching the phone, which would then allow them to assess if they would go into the phone to read the full message. This reflects the resolution of conflicting tasks through assigning priority to one task over the other; both additional factors included in the PARRC model of distraction (Parnell et al. 2016). Reading the first few lines of the text would allow the driver to decide the priority of the text; however, it would also increase the length of time that they would need to take their eyes away from the road in contrast to reading just the name (Harvey and Stanton 2013). This reflects how mobile manufacturers may be influencing the drivers' diversion of attention away from the road. Parnell et al. (2017a) highlight the issue of prohibiting the driver from interacting with the mobile phone, but permitting the mobile phone to interact with the driver.

\subsubsection{Road type}

The finding that drivers were approximately three times more willing to engage with a task on the motorway and A road than at roundabouts supports previous findings that suggest drivers are less willing to engage in other tasks while performing manoeuvres (Lerner et al. 2008; Tivesten and Dozza 2015). Whist drivers seemed indifferent in their intention to engage on A roads or motorways, the relationship between the task and the infrastructure at roundabouts was mentioned frequently when drivers were at roundabouts. The large amount of processing that was required to navigate the roundabout was mentioned by many participants (e.g., "At the moment, no because were on a junction. There is a lot going on. There is things in all the different lanes. And if it was any more than just one press, that would be too much for me to do", Participant 4). This suggests that drivers are able to assess when they are unable to engage with technological tasks while driving and avoid doing so at complex junctions, providing further evidence for their strategic adaption of task engagement.

While drivers were more likely to engage on motorways and A roads, they did vocalise features of these environments that would influence their decision to engage. On the A road, drivers were notably more concerned with the presence of other road users including pedestrians who may step out in the road and other cars who may act unpredictably. On the motorway, drivers made multiple references to the other cars around them, the lane of traffic that they were in and how this would influence their decision to engage, with many stating that they would pull into the slow lane if they needed to engage in a non-driving task while driving. There was also a number of references to lorries and large vehicles that they needed to overtake on the motorway that influenced their decision. This suggests that the drivers did incorporate the surrounding environment into their strategic planning of secondary task engagement. Furthermore, not only was strategic intention evident across road types but also within them. The role of road and infrastructure design is thus revealed in the drivers' decision to engage with distractions. Furthermore, the strategic engagement that is referenced suggests that the drivers' engagement in distracting tasks is not as simple as whether they do engage or they do not engage with certain tasks, as is often studied in surveys (e.g., McEvoy et al. 2006), and previous experimental studies (Metz et al. 2011; Horrey and Lesch 2009; Tivesten and Dozza 2015). The verbal reports evidenced the presence of multiple causal factors that related to the task and road types they were exposed to.

\subsection{Using verbal protocol to capture naturalistic decision making}

The use of verbal protocols aimed to determine drivers' intention to engage, rather than assessing what happens to driving performance when the driver becomes distracted. Understanding the decisions that drivers made and the reasoning for their decisions can inform effective mitigation strategies that can target the underlying reasons distraction occurs, rather that penalising it once it has happened. Verbal protocol (VP) is a simple and low-cost way of collecting data about, and analysing, cognitive processes (Ericsson and Simon 1993). This had obvious advantages to the methodology that could be used and the settings within which it could be measured. The assessment of intention to engage, rather than making the driver physically engage, mitigated some of the safety concerns of studying the behaviour in the real world. Furthermore, Salmon et al. (2017) found that the use of verbal protocols in the real-world driving setting 
may actually enhance driving performance and safety, with driving speed or control unaffected and breaking/acceleration at junctions improving (Salmon et al. 2017). These findings, however, relate to the continuous verbal reports of drivers to assess the drivers' situational awareness, rather than assessing their decision-making process. Therefore, to assess the impact of providing verbal reports, speed metrics were compared during periods when drivers were providing verbal reports (stating their intention to engage in response to the scenarios) and when they were driving without providing verbal intentions. While speed metrics such as average speed and speed variability can infer distraction (e.g., Horrey and Wickens 2004; Burns et al. 2002), they are not perfect predictors and may be influenced by other aspects of the road environment and other road users. Yet, where alternative metrics such as headway cannot be obtained, as was the case in this study, they may offer some insights into driver performance (Kircher and Ahlstrom 2010). Caution, however, is advised in the interpretation of these findings due to the small sample size.

Nonetheless, subjective workload was found to be significantly higher when drivers were verbalising their intention to engage in non-driving tasks in the simulator compared to when doing so on the road. This is in contrast to others who have reported similar workloads on the road and in the simulator (Cantin et al. 2009; Patten et al. 2006). The debrief interviews conducted with drivers after the experimental trials revealed that many found the simulator condition difficult due to a lack of awareness of speed. Particular reference was given to roundabouts, which can be difficult to simulate and can heighten motion sickness (Bittner et al. 2002). These factors of the simulator experience may have influenced these finding. Furthermore, participants were only given a practise drive of approximately $10 \mathrm{~min}$ in the simulator before the experiment. Extensive training in the simulator environment may have reduced workload. Yet, despite increased workload participants were able to maintain the same mean speed when providing verbal reports as when they were driving without verbalising their intention on the A road and motorway. Speed variability, however, was found to differ when driving on the motorway in the simulator, with increased variability when stating their intention to engage. This may suggest that drivers verbal reports may have influenced their performance in the driving task at this time. One explanation for this may be due to the reduced perception of speed attributed to the motorway environment and simulator setting mentioned in the debrief interview. It may also be due to a reduced risk that accompanies simulation research (Bella 2008) that led drivers to limit their investment in managing their speed consistently while also verbalising their intention. Indeed, some participants commented that they felt it hard to infer what their driving speed was in the simulator condition. Conversely, in the real-world condition, where the risk of accident is greater, a difference between mean speed when driving with verbal reports and without verbal reports on the A road was found. A roads led to frequent references to other road users, including other vehicles, pedestrians and cyclists. The complexity and increased risk of accident that this may have posed to participants in the road condition may have led drivers to adapt their speed when verbalising their intention. Adaption at this control level has been found in other complex driving situations with drivers adapting their speed when engaging with secondary tasks to compensate for their reduced performance (e.g., Alm and Nilsson 1994; Strayer et al. 2003; Tivesten and Dozza 2015; Metz et al. 2015).

\subsection{Future research}

This research has established that high fidelity driving simulators can facilitate the assessment of naturalistic decision making to a similar degree as that conducted on the road. Therefore, future research should seek to develop understanding of the drivers' decision to engage with distractions, as this is currently an under-researched area. Furthermore, research is required to determine how VP relates to driving speed and vehicle control on the road and in the simulator. This requires the use of a larger sample of drivers and the comparison of driving while stating intention to be compared along the same road segments as a control drive when no VP is performed. The addition of steering and breaking metrics would assist in the interpretation of this data but was unable to be collected within this research study.

The smaller sample size is a limitation of this study, quantitative studies conducted in simulators have afforded larger sample sizes (e.g., Horberry et al. 2006, $n=31$; Jahn et al. $2005, n=49$ ). Yet, this research has taken an initial look at the potential comparisons that can be drawn from the simulated environment that is commonly relied upon in driver distraction research and real-world driving conditions. While the sample were of a range of ages and comprised of regular and experienced driver, the generalisations that can be made from this limited sample are restricted. Further research to needed to assess the validity of these findings and their generalisations to larger samples. Determining if the findings presented in Tables 11 and 12 apply to a broader sample is of particular interest to assess the potential impact it could have on manufacturers and designers.

Furthermore, continuing with the work of Metz et al (2011) as well as Schömig and Metz (2013) who used eye tracking metrics to inform drivers initiation of secondary tasks, eye tracking metrics could be used to determine what the drivers are monitoring when they are making the decision to engage with secondary tasks. This may show differences is aspects of the road scene that they monitor across road types or task types. Assessment of this in the simulator 
and the road setting would build on the findings of this study to determine how the research setting may influence the drivers' intention to engage and their decision-making process. It would also be of interest to assess how drivers may perform if they were asked to actually complete the tasks. This would assess whether the drivers' perceptions about their ability to complete the task were accurate.

Finally, the study did not allow drivers to use ADAS features that aim to enhance driving performance by automating and assisting with aspects of the driving task. Such features are now common place in modern vehicles and are utilised by many drivers, this, therefore, may limit the generalisations of this research. Others have found that ADAS features can lead to internal dissonance (Vanderhaegen 2017) and features such as adaptive cruise control and forward collision warning systems can actually increase the likelihood that drivers will engage with secondary tasks (Strayer et al. 2003). The influence of ADAS functionality on the drivers verbalised decision-making process is an interesting avenue for future research.

\section{Conclusion}

The decision to engage with secondary tasks has been under-researched in previous years, with the active role of the driver and the surrounding sociotechnical system in the management of the interaction with secondary tasks often ignored. This is despite evidence to suggest that the majority of distractions are voluntarily engaged with (e.g., Beanland et al. 2013). As technologies develop, in line with consumer demand, the factors that influence the decision to engage with tasks that may pose as distractions is important to consider. This study has shown that the drivers decision to engage with technological tasks in the simulator is representative of their decision on the road. Furthermore, the use of verbal protocol methodology can be used to assess the complex interacting factors that are influencing their decision-making process when planning to engage with technological tasks. This includes, the task itself, road infrastructure, the wider context, other road users and the driver. It has been identified how these factors can positively and/ or negatively impact on driver decision making, and should, therefore, be considered by manufacturers to acknowledge the implications of the devices that they develop to be used in the vehicle. Furthermore, this study has provided evidence that drivers do strategically plan their engagement with technological tasks with respect to task type and road type.

Acknowledgements This research was funded by the Engineering and Physical Science Research Council (EPSRC) grant EP/G036896/1 under the Industry Doctoral Training Centre in Transport and the Environment. The authors would like to thank Dr. Alexander Eriksson for his assistance with conducting the on road trials and Dr. Craig Allison and James Brown for their assistance with the simulation development.

Open Access This article is distributed under the terms of the Creative Commons Attribution 4.0 International License (http://creativeco mmons.org/licenses/by/4.0/), which permits unrestricted use, distribution, and reproduction in any medium, provided you give appropriate credit to the original author(s) and the source, provide a link to the Creative Commons license, and indicate if changes were made.

\section{Appendix}

Table 11 presents the key factors increasing or decreasing drivers' engagement with different tasks and Table 12 presents the key factors increasing or decreasing drivers' engagement across road types. 
Table 11 Summary of key factors increasing and decreasing drivers' engagement with different tasks

Task scenario

Text

\section{Context}

Expecting a text

The importance of the message

Task

Phone placed in line of sight, e.g., on dashboard

Notifications flashes up on screen (reflex reaction)

Read first lines to determine urgency/who its from

Single touch/Easily accessible

Place phone on lap

Gradual glances away from road

Short texts

Touch unlock (easier than pin)
Call

\section{Context}

Importance of the phone call

Task

Connect to hand-free/Speaker

Voice dial

Small glances to scroll phone book

If phone is in line of sight

Thumb print phone unlock

Number saved to contacts

If single button-press

Quick and easy to achieve

If already connected, would continue to talk (even in high demand areas, where call may not be initiated)
Factors reducing engagement

\section{Context}

Not urgent enough

Driver

Doesn't need immediate action

Place phone out of sight

Would not use phone at all in vehicle

\section{Task}

Needing to touch the phone

Phone placed out of sight

Need to enter phone pin code

Long messages

If scrolling is involved

Not enough time to read it

Destination Song

Task

Enter in stages (glance back to road)

Use voice input

Use remembered destinations within the system

Use voice entry

Task

Don't need to look

Know where the buttons are

Easy to take one hand off the steering wheel

Very quick and easy to do safely

Its an automatic action

Use steering wheel buttons

Use pre-set buttons

Single button presses

Only one quick glance

Don't have to take eyes off road

Don't like bad songs

Know where the buttons are

Don't have to think about it

Driver

Never use phone while driving

Never going to be important enough

Not capable of doing task

It can wait

Task

Lengthy menus to scroll through

Unlocking the phone

Manual/full number entry

Scrolling through is too complex

Need hands on the wheel

\section{Driver}

Don't want to take hands off the wheel

Need glasses to read the device

Task

Poorly designed feature

Too long/eyes off road for too long

Too many key presses

Complex interaction (more than 1 press)
Driver

Not even think-

ing about it

Task

Too much faff to change, easier to turn it off

If listening through phone - need to unlock phone

Need hands on the steering wheel

If have to go into menus 
Table 12 Summary of key factors increasing and decreasing drivers' engagement across different road types

Task scenario

\begin{tabular}{|c|c|c|}
\hline Motorway & A road & Roundabout \\
\hline \multicolumn{3}{|l|}{ Factors increasing engagement } \\
\hline $\begin{array}{l}\text { Context } \\
\text { Familiarity with the road } \\
\text { On the road for a long time } \\
\text { low traffic levels } \\
\text { Infrastructure } \\
\text { Can see far ahead } \\
\text { Stable/predictable environment } \\
\text { Consistent speed } \\
\text { Low risk environment } \\
\text { Move to slow lane } \\
\text { Straight segments of road } \\
\text { Limited attention required to road } \\
\text { Other road users } \\
\text { Increase distance to lead vehicles } \\
\text { Low levels of traffic around } \\
\text { Road uses keeping their trajectory }\end{array}$ & $\begin{array}{l}\text { Context } \\
\text { Quiet road } \\
\text { Familiarity with the road } \\
\text { Going slowly } \\
\text { Infrastructure } \\
\text { Low demand road } \\
\text { Straight road/good visibility } \\
\text { Slow stop/start traffic } \\
\text { Boring road } \\
\text { Rural areas/less built up } \\
\text { Wide road } \\
\text { Can't stop/pull over } \\
\text { No junctions } \\
\text { Other road users } \\
\text { Predictable road users } \\
\text { Leave gap to vehicle in front }\end{array}$ & $\begin{array}{l}\text { Infrastructure } \\
\text { Stopped, e.g., at light, with handbrake on } \\
\text { During waiting zone before the roundabout } \\
\text { If a queue at the roundabout } \\
\text { Go around roundabout again to get caught by a light } \\
\text { and enter destination } \\
\text { Straighter sections of the roundabout }\end{array}$ \\
\hline \multicolumn{3}{|l|}{ Factors reducing engagement } \\
\hline $\begin{array}{l}\text { Infrastructure } \\
\text { Need to check lanes a lot } \\
\text { Approaching a junction } \\
\text { Bends and obscured line of sight } \\
\text { Things happen quickly } \\
\text { Changing lanes } \\
\text { Can't pull over on the motorway } \\
\text { Other road users } \\
\text { Large vehicles around } \\
\text { Lots of traffic } \\
\text { Fast traffic from behind }\end{array}$ & $\begin{array}{l}\text { Infrastructure } \\
\text { Residential/built up areas } \\
\text { Junctions with merging traffic } \\
\text { Opportunity to pull over } \\
\text { Wait for a red light } \\
\text { Small/narrow road } \\
\text { If turning/manoeuvring the vehicle } \\
\text { Windy roads } \\
\text { Wait until after a bend } \\
\text { Other road users } \\
\text { Near cyclists/pedestrians } \\
\text { Lots of oncoming traffic } \\
\text { Parked cars, watch for pedestrians } \\
\text { Lorry on the road } \\
\text { Erratic driver ahead } \\
\text { Pedestrians may see you using the device } \\
\text { (anti-social) }\end{array}$ & $\begin{array}{l}\text { Context } \\
\text { Don't know where the exit is/not familiar } \\
\text { Driving demand is too high } \\
\text { Infrastructure } \\
\text { Wait until after the roundabout } \\
\text { Big manoeuvre/too much steering } \\
\text { Needs a lot of attention, especially trying to pull out } \\
\text { Need to change lanes } \\
\text { Unpredictable road environment } \\
\text { Good judgement needed to enter roundabout } \\
\text { Blind spots } \\
\text { Concentration to stay in the right lane } \\
\text { Other road users } \\
\text { Looking for oncoming cars/hazards } \\
\text { Lots of cars changing lanes } \\
\text { Other traffic monitoring }\end{array}$ \\
\hline
\end{tabular}

\section{References}

Allison CK, Parnell KJ, Brown JW, Stanton NA (2017) Modelling the real world using STISIM drive ${ }^{\circledR}$ simulation software: a study contrasting high and low locality simulations. In: International conference on applied human factors and ergonomics. Springer, Cham, pp 906-915

Alm H, Nilsson L (1994) Changes in driver behaviour as a function of handsfree mobile phones - a simulator study. Accid Anal Prev 26(4):441-451

Banks VA, Stanton NA (2015) Contrasting models of driver behaviour in emergencies using retrospective verbalisations and network analysis. Ergonomics 58(8):1337-1346

Beanland V, Fitzharris M, Young KL, Lenné MG (2013) Driver inattention and driver distraction in serious casualty crashes: data from the Australian National crash in-depth study. Accid Anal Prev 54:99-107

Bella F (2008) Driving simulator for speed research on two-lane rural roads. Accid Anal Prev 40(3):1078-1087

Bittner A, Simsek Jr O, Levison W, Campbell J (2002) On-road versus simulator data in driver model development driver performance model experience. Transp Res Rec J Transp Res Board 1803:38-44

Boer ER, Hoedemaeker M (1998) Modeling driver behavior with different degrees of automation: a hierarchical decision framework of interacting mental models. In: Conference on human decision making and manual control, Valenciennes, December. LAMIH

Brookhuis KA, de Vries G, de Waard D (1991) The effects of mobile telephoning on driving performance. Accid Anal Prev 23(4):309-316

Brown ID, Tickner AH, Simmonds DC (1969) Interference between concurrent tasks of driving and telephoning. J Appl Psychol 53(5):419-424

Burnett GE (2008) Designing and evaluating in-car user-interfaces. In: Lumsden $\mathbf{J}$ (ed) Handbook of research on user-interface design and evaluation for mobile technology. Idea Group Inc, Hershey

Burns PC, Parkes A, Burton S, Smith RK, Burch D (2002) How dangerous is driving with a mobile phone? Benchmarking the impairment to alcohol. In: TRL report TRL547. TRL Limited, Berkshire

Cantin V, Lavallière M, Simoneau M, Teasdale N (2009) Mental workload when driving in a simulator: effects of age and driving complexity. Accid Anal Prev 41(4):763-771 
Carsten O, Kircher K, Jamson S (2013) Vehicle-based studies of driving in the real world: the hard truth? Accid Anal Prev 58:162-174

Dekker SWA (2002) Reconstructing human contributions to accidents: the new view on error and performance. J Saf Res 33(3):371-385

Department for Transport (2015) Reported road casualties great Britain: 2015. Annual Report. https://www.gov.uk/government/uploa ds/system/uploads/attachment_data/file/568484/rrcgb-2015.pdf Accessed 28 Apr 2017

Dingus TA, Klauer SG, Neale VL, Petersen A, Lee SE, Sudweeks JD et al (2006) The 100-car naturalistic driving study, Phase II-results of the 100-car field experiment (no. HS-810 593). Washington USA

Dogan E, Steg L, Delhomme P (2011) The influence of multiple goals on driving behavior: the case of safety, time saving, and fuel saving. Accid Anal Prev 43(5):1635-1643

Ericsson KA, Simon HA (1993) Protocol analysis. MIT, Cambridge

Eriksson A, Banks VA, Stanton NA (2017) Transition to manual: comparing simulator with on-road control transitions. Accid Anal Prev 102:227-234

Gordon SE, Gill RT (1997) Cognitive task analysis. In: Zsambok C, Klein G (eds) Naturalistic decision making. Laurence Erlbaum Associates, Mahwah, pp 131-140

Haigney DE, Taylor RG, Westerman SJ (2000) Concurrent mobile (cellular) phone use and driving performance: task demand characteristics and compensatory processes. Transp Res Part F Traffic Psychol Behav 3(3):113-121

Hart SG, Staveland LE (1988) Development of NASA-TLX (Task Load Index): results of empirical and theoretical research. Adv Psychol $52: 139-183$

Harvey C, Stanton NA (2013) Usability evaluation for in-vehicle systems. CRC, Boca Reta

Harvey C, Stanton NA, Pickering CA, McDonald M, Zheng P (2011) Context of use as a factor in determining the usability of in-vehicle devices. Theor Issues Ergon Sci 12(4):318-338

Holtzblatt K, Jones. S (1993) Contextual inquiry: a participatory technique for system design. In: eds. Schuler D, Aki N (eds) Participatory design: principles and practices. Lawrence Erlbaum, New Jersey, pp 177-210

Horberry T, Anderson J, Regan MA, Triggs TJ, Brown J (2006) Driver distraction: the effects of concurrent in-vehicle tasks, road environment complexity and age on driving performance. Accid Anal Prev 38(1):185-191

Horrey WJ, Lesch MF (2009) Driver-initiated distractions: examining strategic adaptation for in-vehicle task initiation. Accid Anal Prev 41(1):115-122

Horrey WJ, Wickens CD (2004) Driving and side task performance: the effects of display clutter, separation, and modality. Hum Fact J Hum Fact Ergonom Soc 46(4):611-624

Isenberg DJ (1986) Thinking and managing: a verbal protocol analysis of managerial problem solving. Acad Manag J 29(4):775-788

Jahn G, Oehme A, Krems JF, Gelau C (2005) Peripheral detection as a workload measure in driving: effects of traffic complexity and route guidance system use in a driving study. Transp Res Part F Traffic Psychol Behav 8(3):255-275

Kahneman D, Slovic P, Tversky A (1982) Judgment under uncertainty: heuristics and biases. Cambridge University Press, New York, USA

Kircher K, Ahlstrom C (2010) Predicting visual distraction using driving performance data. Ann Adv Automot Med 54:333-342

Kircher K, Ahlstrom C (2017) Minimum required attention: a humancentered approach to driver inattention. Hum Fact J Hum Fact Ergonom Soc 59(3):471-484

Kircher A, Vogel K, Tornos J, Bolling A, Nilsson L, Patten C, Malmstrom T, Ceco C (2004) Mobile telephone simulator study. Swedish National Road and Transport Research Institute, Linkoping
Klein GA (1989) Recognition-primed decisions. In: Rouse W (ed) Advances in man-machine systems research. JAI, Greenwich, pp 47-92

Klein G (2008) Naturalistic decision making. Hum Fact J Hum Fact Ergonom Soc 50(3):456-460

Klein GA, Calderwood R, Clinton-Cirocco A (1986) Rapid decision making on the fire ground. In: Proceedings of the human factors society annual meeting, vol 30, issue 6. SAGE, Los Angeles, pp $576-580$

Lansdown TC (2012) Individual differences and propensity to engage with in-vehicle distractions-A self-report survey. Transp Res Part F Traffic Psychol Behav 15(1):1-8

Lansdown TC, Brook-Carter N, Kersloot T (2004) Distraction from multiple in-vehicle secondary tasks: vehicle performance and mental workload implications. Ergonomics 47(1):91-104

Lansdown TC, Stephens AN, Walker GH (2015) Multiple driver distractions: a systemic transport problem. Accid Anal Prev 74:360-367

Larsson P, Dekker SW, Tingvall C (2010) The need for a systems theory approach to road safety. Saf Sci 48(9):1167-1174

Lee JD (2014) Dynamics of driver distraction: the process of engaging and disengaging. Ann Adv Automot Med 58:24

Lee JD, Strayer DL (2004) Preface to the special section on driver distraction. Hum Fact J Hum Fact Ergonom Soc 46(4):583-586

Lerner N (2005) Deciding to be distracted. In: Proceedings of the third international driving symposium on human factors in driver assessment, training and vehicle design. June. Maine, USA, pp 499-505

Lerner N, Singer J, Huey R (2008) Driver strategies for engaging in distracting tasks using in-vehicle technologies (no. DOT HS 810 919). National Highway Traffic Safety Administration, Washington, DC

Matthews BW (1975) Comparison of the predicted and observed secondary structure of T4 phage lysozyme. BBA Protein Struct 405(2):442-451

McEvoy SP, Stevenson MR, Woodward M (2006) The impact of driver distraction on road safety: results from a representative survey in two Australian states. Inj Prev 12(4):242-247

Metz B, Schömig N, Krüger HP (2011) Attention during visual secondary tasks in driving: adaptation to the demands of the driving task. Transp Res Part F Traffic Psychol Behav 14(5):369-380

Metz B, Landau A, Hargutt V (2015) Frequency and impact of handsfree telephoning while driving - results from naturalistic driving data. Transp Res Part F Traffic Psychol Behav 29(0):1-13

Neale VL, Dingus TA, Klauer SG, Sudweeks J, Goodman M (2005) An overview of the 100-car naturalistic study and findings. National Highway Traffic Safety Administration, Paper 05-0400, USA

Parnell KJ, Stanton NA, Plant KL (2016) Exploring the mechanisms of distraction from in-vehicle technology: the development of the PARRC model. Saf Sci 87:25-37

Parnell KJ, Stanton NA, Plant KL (2017a) Understanding drivers' strategies for engaging with in-vehicle technology while driving: an interview study. In: Presentation given at the 5th international conference on driver distraction and inattention. March. Paris, France

Parnell KJ, Stanton NA, Plant KL (2017b) What's the law got to do with it? Legislation regarding in-vehicle technology use and its impact on driver distraction. Accid Anal Prev 100:1-14

Patten CJ, Kircher A, Östlund J, Nilsson L, Svenson O (2006) Driver experience and cognitive workload in different traffic environments. Accid Anal Prev 38(5):887-894

Pedic F, Ezrakhovich A (1999) A literature review: the content characteristics of effective VMS. Road Transp Res 8(2):3

Phillips JK, Klein G, Sieck WR (2004) Expertise in judgment and decision making: a case for training intuitive decision skills. In Eds 
Koehler DJ, Harvey N (eds). Blackwell handbook of judgment and decision making. Wiley, Oxford, pp 297-315

RAC (2016) RAC Report on motoring 2016. The road to the future. https://www.rac.co.uk/pdfs/report-on-motoring/rac-report-onmotoring-2016-outline.pdf Accessed 28 Apr 2016

Rakauskas ME, Gugerty LJ, Ward NJ (2004) Effects of naturalistic cell phone conversations on driving performance. J Saf Res 35(4):453-464

Ranney TA, Harbluk JL, Noy YI (2005) Effects of voice technology on test track driving performance: implications for driver distraction. Hum Fact J Hum Fact Ergonom Soc 47(2):439-454

Reason J (1990) Human error. Cambridge University Press, Cambridge

Regan MA, Lee JD, Young K (2008) Driver distraction: theory, effects, and mitigation. CRC, Boca Raton

Reimer B (2009) Impact of cognitive task complexity on drivers' visual tunnelling. Transp Res Rec J Transp Res Board 2138:13-19

Salmon PM, McClure R, Stanton NA (2012) Road transport in drift? Applying contemporary systems thinking to road safety. Saf Sci 50(9):1829-1838

Salmon PM, Goode N, Spiertz A, Thomas M, Grant E, Clacy A (2017) Is it really good to talk? Testing the impact of providing concurrent verbal protocols on driving performance. Ergonomics 60(6):770-779

Schömig N, Metz B (2013) Three levels of situation awareness in driving with secondary tasks. Saf Sci 56:44-51

Sharples S, Shalloe S, Burnett G, Crundall D (2016) Journey decision making: the influence on drivers of dynamic information presented on variable message signs. Cognit Technol Work 18(2):303-317

Simon H (1957) Models of man: social and rational. Wiley, New York

Stanton NA, Young MS (1999) What price ergonomics? Nature 399:197-198

Strayer DL, Drews FA, Johnston WA (2003) Cell phone-induced failures of visual attention during simulated driving. J Exp Psychol Appl 9(1):23

Stutts JC, Reinfurt DW, Staplin L, Rodgman EA (2001) The role of driver distraction in traffic crashes. AAA Foundation for Traffic Safety, Washington, DC

Summala H (2007) Towards understanding motivational and emotional factors in driver behaviour: comfort through satisficing. In: Cacciabue $\mathrm{C}$ (ed) Modelling driver behaviour in automotive environments. Springer, New York, pp 189-207

Summala H, Lamble D, Laakso M (1998) Driving experience and perception of the lead car's braking when looking at in-car targets. Accid Anal Prev 30(4):401-407
Tingvall C, Ekstein L, Hammer M (2009) Government and industry perspectives on driver distraction. In Regan MA, Lee JD, Young KL (eds) Driver distraction: theory, effects and mitigation. CRC Press, Florida, USA

Tivesten E, Dozza M (2015) Driving context influences drivers' decision to engage in visual-manual phone tasks: evidence from a naturalistic driving study. J Saf Res 53:87-96

Tractinsky N, Ram ES, Shinar D (2013) To call or not to call-that is the question (while driving). Accid Anal Prev 56:59-70

Tsimhoni O, Smith D, Green P (2004) Address entry while driving: speech recognition versus a touch-screen keyboard. Hum Fact $\mathbf{J}$ Hum Fact Ergonom Soc 46:600-610

Vanderhaegen F (2017) Towards increased systems resilience: new challenges based on dissonance control for human reliability in cyber-physical and human systems. Annu Rev Control 44:316-322

Walker GH, Stanton NA, Chowdhury I (2013) Self-Explaining Roads and situation awareness. Saf Sci 56:18-28

Wickens CD (2002) Multiple resources and performance prediction. Theor Issues Ergonom Sci 3(2):159-177

World Health Organization (2011) Mobile phone use: a growing problem of driver distraction. http://apps.who.int/iris/bitstream/10665 /44494/1/9789241500890_eng.pdf. Accessed 05 Sep 2017

Young KL, Lenné MG (2010) Driver engagement in distracting activities and the strategies used to minimise risk. Saf Sci 48(3):326-332

Young KL, Regan M (2007) Driver distraction: a review of the literature. In: Faulks IJ, Regan M, Stevenson M, Brown J, Porter A, Irwin JD (eds) Distracted driving. Australasian College of Road Safety, Sydney, pp 379-405

Young KL, Salmon PM (2015) Sharing the responsibility for driver distraction across road transport systems: a systems approach to the management of distracted driving. Accid Anal Prev 74:350-359

Young MS, Stanton NA (2002) Malleable attentional resources theory: a new explanation for the effects of mental underload on performance. Human factors 44(3):365-375

Young MS, Stanton NA (2004) Taking the load off: investigations of how adaptive cruise control affects mental workload. Ergonomics 47(9):1014-1035

Young KL, Salmon PM, Cornelissen M (2013) Missing links? The effects of distraction on driver situation awareness. Saf Sci $56: 36-43$ 